\title{
Walking mechanism design of intelligent spraying robot
}

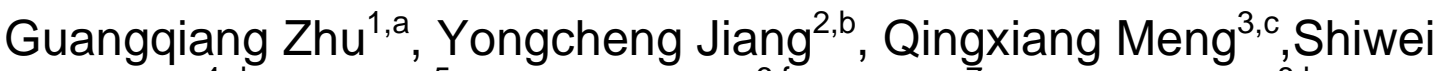 Zhu $^{4, \mathrm{~d}}$, Meaza ${ }^{5, \mathrm{e}}$, Xiuping Hua ${ }^{6, \mathrm{f}}$,Bing $\mathrm{Li}^{7,9}$,Qinglin $\mathrm{Han}^{8, \mathrm{~h}}$}

\author{
${ }^{1}$ College of Mechanical Engineering, Jiamusi University, Jiamusi 154007, China; \\ ${ }^{2}$ College of Mechanical and Electrical Engineering, Northeast Forestry University, Harbin 150040, \\ China. \\ azhuguangqiang123@126.com,,jiangyongcheng@126.com, ${ }^{\mathrm{C}}$ mengqiangxiang714@126.com
}

Keywords: Agricultural traveling mechanism, PROE, Servo drives, Servo motors, Kinematic analysis equation.

\begin{abstract}
In this paper, the walking mechanism model of intelligent spray robot with it is stable and accurate approaches to ward the design structure are introduced. According the requirements of intelligent spraying robot, it uses the following method. First of all, designing the 3D model in PROE, for improving or carried on the simulation analysis of the three wheel chassis walking mechanism. Secondly, the servo drive mode analyzes power source of the walking mechanism and also the servo motor supports to complete the work of walking and walking mechanism of the intelligent robot .Last, calculated the suitable for walking and working demand reduction ratio. This paper mainly introduces the condition of the walking mechanism to satisfy the steering condition and establishes the kinematics equation. The experimental results show that the walking mechanism can fully meet the requirements of intelligent spraying robot, for the development of the whole intelligent spraying robot sequential to lay a solid basis.
\end{abstract}

\section{Introduction}

Since there are a large number of labor rural into cites of china that causes the labor rural for becoming more prominent, the urgent need for intelligent farm machinery to transform traditional agricultural production, in response to regional climatic conditions, natural disasters and pests prevention and control, are inseparable form intelligent agricultural machinery. Currently our country is developing rapidly for using intelligent agricultural machinery[1], but it is not applicable available in different places of the geographical and environment of the rural to used agricultural machinery, uses backward of technology, the main reason for the above problem us that agricultural crops are growth different around the soil and also in it is difficult to use the agricultural machineries in a complex the geographical and environment. At present, the domestic spraying operation, basically using the traditional vehicle-mounted spraying manner, which not only increased the operator labor intensity, low work efficiency, but also enable the operator exposure to pesticide spraying environment, harm their health, and uneven spraying, there is a residual. In order to eliminate these disadvantages, it is necessary to develop the spraying robot to control the pests and diseases. In this paper a king of intelligent spraying robot with it is waking mechanism designed, that can upgrade and change the traditional spraying equipment and also methods[2 $\sim 5]$.

\section{Structure Design and Parameter}

The walking mechanism adopts a three wheel drive chassis, the source of power is servo drive and servo motor, the front wheel uses as a steering motor, where as the remaining left and right wheels uses as a differential steering that helps for achieving the drive mechanism of the three wheels and that has the following features such as: using control the position model, closed-loop control of achieved to speed and torque, to overcome the problem of stepper motor lose step, it is ability strong to resist overload, can bear the load of three times the rated torque, can realize the 
design requirements of the walking mechanism. By systematic comparison, the walking mechanism adopts the wheel hub motor (Panasonic MHMJ082G1V motor) structure, the rated power is 750W.

$$
\frac{\frac{v}{60 \times 2 \times \pi \times r \times n}}{1000}=i
$$

The walking mechanism belongs relates to the servo motor speed the can reaches up to 3000 $\mathrm{r} / \mathrm{min}$,therefore there is a need to use worm and worm wheel reducer in the design part to reduce speed and increase torque. Reducer gear can be divided into two parts that is planetary wheel reduction gear unit and worm type reduction gear unit. When comparing with planetary wheel reducer gear, the worm gear and worm deceleration is more precision and high transmission of power, it is capable of to rotate 90 degree angle drive and improving the torque and efficiency, it has self-locking functioning performance, low manufacturing cost. The calculation formula of reduction ratio is:

In the formula,

$$
\begin{aligned}
& \text { V- traveling speed mechanism }(\mathrm{km} / \mathrm{h}) \text {; } \\
& \mathrm{r} \text { - the wheel radius }(\mathrm{m}) \text {; } \\
& \mathrm{n} \text { - servo motor rated speed ( } \mathrm{r} / \mathrm{min}) \text {. }
\end{aligned}
$$

Because the walking mechanism does not need too much travel speed, so set the running speed $\mathrm{V}$ $=5 \mathrm{~km} / \mathrm{h}$, the wheel radius $0.125 \mathrm{~m}$, the servo motor rated speed $3000 \mathrm{r} / \mathrm{min}$, by equation (1) to calculate the reduction gear ratio 1:30, physical assembly shown in Figure 1.

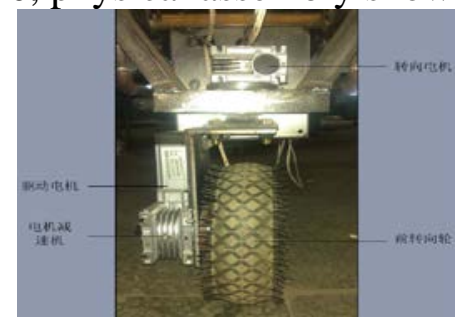

Fig.1 Physical picture of loading speed reducer

\section{Kinematic analysis of the Running gear}

Since the walking mechanism adopts a three wheel drive chassis, the front wheel steering wheel speed will not change that $\mathrm{V}_{0}=5 \mathrm{~km} / \mathrm{h}$, it is assumed that between the wheels there is only a rolling friction that force balance the walking mechanism, so when the front steering runs, you just changed differential speed of the left and right wheels. Set as the three wheel servo motor speed as $\mathrm{n}_{0}, \mathrm{n}_{1}$, and $\mathrm{n}_{2}$, respectively having units is $\mathrm{r} / \mathrm{min}$, it has a transmission ratio of (i), as shown in the following formula:

$$
\begin{aligned}
& \omega_{0}=\frac{\pi n_{0}}{30 i} \\
& \omega_{1}=\frac{\pi n_{1}}{30 i} \\
& \omega_{2}=\frac{\pi n_{2}}{30 i}
\end{aligned}
$$

Seen by the rigid body kinematics formula is as follows:

$$
v=r \times \omega(5)
$$

In the formula:

$$
\begin{aligned}
& \text { V- Traveling speed mechanism (km / h); } \\
& \mathrm{r} \text { - The wheel radius }(\mathrm{m}) \text {; } \\
& \omega \text { - Wheel angular velocity (rad / s); }
\end{aligned}
$$

When walking mechanism steering, since the front steering wheel deflection angle a, so it will produce a front wheel center line and the center line of the back of the intersection points, take this intersection point as the origin, and with the front wheel and the left and right rear wheel distance as 
the radius of the formation of three concentric circles, as shown in Figure2. The length $(\mathrm{k})$ of the chassis and the width (2L) can calculate the radius of three wheel circles as $R_{0}, R_{1}$ and $R_{2}$ respectively that describes in the formula below:

$$
\begin{aligned}
& R_{0}=\frac{K}{\sin \alpha} \\
& R_{1}=\frac{K}{\tan \alpha}-L \\
& R_{2}=\frac{K}{\tan \alpha}+L
\end{aligned}
$$

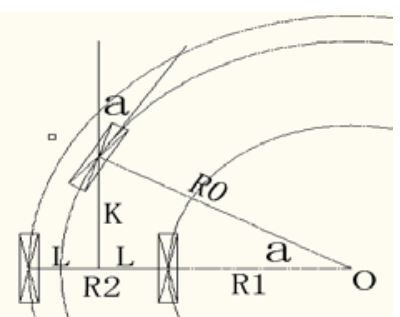

Fig.2 Analysis schematic Steering

The corners of each wheel correspond to a circle, assuming the completion of concentric half the circumference of a circle, computation formula is as follows:

$$
c=\pi \times R
$$

So it is obvious to calculate half of the circumference of the three concentric circles respectively is $\mathrm{C}_{0}, \mathrm{C}_{1}$ and $\mathrm{C}_{2}$. Since as we have taken the same time $(\mathrm{t})$ for each of three wheel steering, so can work out the instantaneous speed of the walking mechanism that can be shown in the following formula:

$$
v=\frac{c}{t}
$$

Can calculate the speed of the three wheels were $\mathrm{V}_{0}, \mathrm{~V}_{1}$ and $\mathrm{V}_{2}$.

By equation (2) to (10) can be obtained and the relationship between the rotational speeds of the rotation angle, computation formula is as follows:

$$
\begin{aligned}
& \boldsymbol{n}_{\mathbf{O}}=\frac{150 \boldsymbol{i}}{\pi r} \\
& n_{1}=\frac{150 i\left(\frac{k}{\tan \alpha}-L\right)}{\frac{\pi r k}{\sin \alpha}} \\
& n_{2}=\frac{150 i\left(\frac{k}{\tan \alpha}+L\right)}{\frac{\pi r k}{\sin \alpha}}
\end{aligned}
$$

By the formula (11) to (13) analysis concluded, when $n_{0}=n_{1}=n_{2}$, namely $a=0^{\circ}$, the three wheels speed is the same, the walking mechanism to do rectilinear motion. When $\mathrm{n}_{0} \neq \mathrm{n}_{1} \neq \mathrm{n}_{2}$ that $a \neq 0$, the front wheels turn a certain angle, the three wheel speed changes, equal to the angle alpha of concentric circles corresponding formula of the speed, the walking mechanism do steering motion. By the above analysis shows that only when the front wheel steering, three of the wheel speed changes, and can according to the steering Angle can be calculated each wheel speed, can realize the steering and linear motion, thus verified the correctness and feasibility of the walking mechanism.

\section{Summary}

Designing of the walking mechanism of a spraying robot, it is description and analysis of the walking mechanism of the organization and the principle of work, establish the spraying robot walking mechanism of three dimensional models, and analyzing its kinematics. The results show that the feasibility of the walking mechanism scheme, and has certain practicability, intelligent 
spraying mechanism overall design research has laid a solid foundation.

\section{Acknowledgement}

Jiamusi University graduate students of science and technology innovation projects(LZZ2015_002), (LZZ2014_007).

Jiamusi University science and technology innovation team (Cxtd-2013-01).

Scientific Research Foundation for the Returned Overseas Chinese Scholars, State Education Ministry (20141685).

\section{References}

[1]. Qigao Feng, Hanping Mao. The Development Present Situation and Countermeasure on Agricultural MechanizationinChina. [J]. Agricultural Mechanization Research. (2010)No.2, p.245-248.

[2]. Zonghao Liu, Caihong Shi, Shaohua Kang. Design and Obstacle Analysis of a Novel Agricultural Machinery Walking Mechanism. [J].Agricultural Mechanization Research. (2014) No.7, p.220-224.

[3]. Xue Sun, Ming Song, Minghui Yao. Innovative Design of Orchard Spraying Robot. [J]. Anhui Agricultural Sciences. Vol.41 (2013)No.30, p.12204-12206.

[4]. Meng Lee,Weilian Sun. The Mechanical Structure Creative Design of Agricultural Spraying Robot. [J]. Agricultural Mechanization Research. (2008) No.1, p.102-105.

[5]. Dajun Zou, Jianjun Zhang,Shouyong Xie. The Moving Mechanism Design of Blueberry Spraying Robot. [J]. Agricultural Mechanization Research. (2009) No.10, p.102-104. 